\title{
Análise das definições de folksonomia: em busca de uma síntese
}

Renato Fernandes Corrêa

Docente do Departamento de Ciência da Informação e do Programa de Pós-Graduação em Ciência da Informação da Universidade Federal de Pernambuco. Doutor em Ciência da Computação pela Universidade Federal de Pernambuco (2008)

Raimunda Fernanda dos Santos

$\begin{aligned} & \text { Concluinte do mestrado em Ciência da } \\ & \text { Informação pela Universidade }\end{aligned}$ Federal de
Pernambuco (2015)

http://dx.doi.org/10.1590/1981-5344/2571

Objetiva criar uma síntese das definições do termo Folksonomia no âmbito da Literatura da Ciência da Informação através de análise dos conceitos relacionados. Visa especificamente contribuir para a consolidação do conceito de Folksonomia levando em conta as definições elencadas pelos autores que trabalham com a temática em questão e as ideias do criador desta terminologia, Thomas Vander Wal. Tem como metodologia a pesquisa exploratória e bibliográfica, como base na análise de conteúdo e em revisão de literatura sobre Folksonomia e representação colaborativa da informação. Destaca que não há ainda uma definição única, clara, bem enunciada e rigorosa acerca da Folksonomia. Conclui conceituando a Folksonomia como o resultado do processo de etiquetagem livre (atribuição de etiquetas ou palavraschave) realizada pelos usuários mediante o emprego de termos provenientes da linguagem natural - dispensando o auxílio de vocabulários controlados - em ambientes digitais colaborativos visando indexar recursos informacionais compartilhados de qualquer formato (textos, imagens, áudio, vídeo, etc.) para fins de sua representação e recuperação.

Palavras-chave: Organização da informação. Representação da Informação. Folksonomia. Indexação social. Etiquetagem. 


\title{
Analysis of folksonomy definitions: in search of a synthesis
}

\begin{abstract}
This work aims to create an overview of the uses of the term folksonomy in the Literature of Information Science through the analysis of the enunciated concepts. It contributes with a step toward consolidation of the folksonomy concept taking into account definitions used by authors working with the theme and by the creator of this terminology, Thomas Vander Wal. The research's nature is exploratory and bibliographical based on content analysis and literature review about folksonomy and collaborative representation of information. It points out that there is not a single, clear, well enunciated and rigorous definition about folksonomy. It concludes proposing the concept of Folksonomy as: the result of free labeling process (assignment of tags or keywords) performed by users through the use of terms from the natural language - dispensing the use of controlled vocabularies - in collaborative digital environments aiming to indexing shared information resources of any format (text, images, audio, video, etc.) for purposes of their representation and retrieval.
\end{abstract}

Keywords: Information organization. Information Representation. Folksonomy. Social indexing. Tagging.

Recebido em 01.10.2015 Aceito em 04.06.2018

\section{Introdução}

O caráter dinâmico e interativo da Web 2.0 gerou novas possibilidades para o desenvolvimento de produtos e serviços que beneficiam a participação ativa dos usuários na coordenação das ações de produção, organização e disseminação de informações no ciberespaço. Diante do crescente aumento na produção de documentos e na participação ativa dos usuários em ambiente digital, surge uma nova forma de organização e representação das informações em que não se adota regras ou políticas de indexação visando o controle do vocabulário empregado para a descrição dos recursos. 
Esta classificação é denominada de Folksonomia e se baseia na filosofia colaborativa, resultante dos preceitos de interatividade da Web 2.0. Ela potencializa a memória coletiva em meio digital por meio das práticas de registrar, organizar e recuperar as informações na Web.

Corrobando com as ideias das autoras Catarino e Baptista (2007) e com base nas pesquisas realizadas na literatura, verifica-se que ainda não há uma conceituação única, clara e rigorosa do real significado da Folksonomia no âmbito da produção científica sobre esta temática na Ciência da Informação, o que pode dificultar o entendimento das suas características, finalidades e possibilidades de aplicação.

Sendo assim, esta pesquisa objetiva criar uma síntese acerca das definições do termo Folksonomia através dos conceitos enunciados. Mais especificamente, objetiva contribuir com um passo na direção à consolidação de uma definição, levando em conta os conceitos extraídos dos trabalhos publicados na área de Ciência da Informação.

Mediante tais considerações, acredita-se que a presente pesquisa é relevante uma vez que contribui com a produção científica sobre a temática em questão na literatura nacional da Ciência da Informação, no que concerne à discussão dos aspectos relativos à conceituação da Folksonomia, possibilitando o entendimento do que é a mesma, assim como dos termos relacionados.

Nesse entendimento, a seguir são discutidas as principais características da Folksonomia, as quais são apontadas na literatura nacional e internacional sobre essa temática. Em seguida, é apresentada a análise e síntese sobre os diversos usos desse termo mediante a extração dos conceitos enunciados em trabalhos nacionais e internacionais da área de Ciência da Informação. $\mathrm{Na}$ oportunidade, também são apresentados sete aspectos que designam os principais elementos presentes nas definições de Folksonomia analisadas. Além disso, levando em consideração as definições extraídas dos trabalhos analisados na pesquisa, é apresentada uma definição para o termo Folksonomia visando contribuir com um passo na direção à consolidação do conceito.

\section{Características da Folksonomia}

Para analisar as definições de Folksonomia na literatura da Ciência da Informação, tornou-se necessário discutir as principais características da mesma.

Mathes (2004), por exemplo, destaca que ela se caracteriza como uma classificação social realizada pelos usuários dos sites ou ferramentas objetivando categorizar os objetos informacionais atribuindo-lhes palavras-chaves. Sendo assim, a natureza coletiva desses termos configura-se como uma característica essencial inerente à Folksonomia para representar o conteúdo de recursos disponíveis na Web em diferentes formatos como textos, livros, artigos, fotografias, vídeos, músicas, links etc. 
Para dar segmento a essa consideração, Wal (2006) afirma que o processo de etiquetagem dos objetos informacionais pode ser descrito em três tipos de elementos:

1) as tags são consideradas como pedaços de informação separados de um objeto informacional, contudo relacionado a ele;

2) importância do claro entendimento do usuário acerca do objeto que está sendo "rotulado";

3) existência de subjetividade na escrita dos descritores, uma vez que a prática evidencia a identidade individual do autor da tag.

Dessa forma, o alto grau de liberdade para a categorização dos recursos informacionais em sistemas colaborativos acentua a descentralização no processo de representação da informação, pois quem classifica o conteúdo são as próprias pessoas interessadas nele, com diversos graus de subjetividade e envolvimento tanto de conhecimento, quanto por experiência ou sentimento. Destarte, tal prática pode implicar em resultados positivos e negativos no que concerne à representação e recuperação da informação em ambientes digitais.

A livre inclusão de metadados por usuários ou grupos de pessoas e a utilização de uma linguagem natural para a representação da informação são características básicas inerentes à Folksonomia, uma vez que ela tem como um de seus elementos a abordagem bottom-up ${ }^{1}$. Desse modo, as tags podem ser baseadas em perspectivas como "assunto, forma, propósito, tempo, tarefa ou status afetivo ou reações críticas e uma miríade de outros motivos" (VIERA; GARRIDO, 2011, p. 8).

Ademais, é importante enfatizar que, devido ao caráter flexível para a atribuição de termos, configura-se também como uma das características da Folksonomia a diversidade de etiquetas em um ou vários idiomas, bem como a existência de conceitos que podem incluir-se em notas de escopo ${ }^{2}$ em instrumentos de controle terminológico.

Nessa concepção, Brandt e Medeiros (2010, p. 120) mencionam acerca do caráter inverso da Folksonomia quando comparada a outros esquemas de representação do conhecimento:

Entende-se que a folksonomia, comparada a outros esquemas de representação do conhecimento, é gerada de forma inversa: primeiro se classificam os objetos informacionais, e, posteriormente, surge uma nova folksonomia, representada visualmente pela tag cloud. Já nas outras ferramentas como as taxonomias e os tesauros, os objetos informacionais são classificados somente quando elas já existem: um tesauro, por exemplo, é usado para a escolha dos termos que irão descrever o conteúdo de um documento.

\footnotetext{
${ }^{1}$ A abordagem bottom-up (em português, de baixo para cima) diz respeito à estrutura que é centrada no usuário para a participação ativa na produção e consumo de informações em ambientes colaborativos.

2 Recurso complementar para vocabulários controlados que objetiva explicitar a amplitude ou o entendimento atribuído a um conceito, bem como determinar o contexto ou as condições em que ele deve ser empregado.
} 
Nesse segmento, González (2006) enfatiza que no processo atribuição de etiquetas para representar os recursos não é necessário construir um conjunto hierárquico de tags, tendo em vista a possibilidade de empregar etiquetas semânticas para efetuar a indexação. Além disso, as etiquetas se apresentam em forma de conjuntos e não de hierarquias.

Assim, a Folksonomia possui um caráter de agregação de etiquetas, o qual propicia a observação de tags relacionadas ao conteúdo em que foi indexado. Essas tags relacionadas são organizadas automaticamente através de aglomerações de etiquetas denominadas tag cloud (ou nuvem de tags) que possibilitam a recuperação da informação.

Para Caldas e Moreira (2009, p. 7-8), a nuvem de tags consiste em um conjunto de etiquetas apresentadas em tamanhos diferentes e os destaques do tamanho que elas são exibidas são designados a partir de critérios específicos do sistema que a utiliza:

Alguns [sistemas] atribuem este privilégio para a frequência com que determinada etiqueta é requisitada na busca de informação, outros de forma mais comum, à frequência com que uma etiqueta é utilizada pelos usuários do sistema. Ou então, um destaque dentro da frequência de utilização das etiquetas por determinado usuário quando a consulta é localizada no conjunto de documentos de um usuário específico [...]. Também através desse recurso o usuário obtém facilidades para visualizar quais as etiquetas são mais usadas em uma comunidade. Isso funciona como uma orientação para futuras postagens de documentos.

Nesse sentido, a nuvem de tags é dinâmica, uma vez que representa um meio de navegação entre um conjunto de documentos existentes em um dado sistema viabilizando o acesso direto aos documentos por meio das etiquetas elencadas nesse cenário. Além disso, é importante acrescentar que uma nuvem de etiquetas não é necessariamente caracterizada pela prática da Folksonomia, tendo em vista que muitas vezes ela é empregada para fins de estética em muitos sites e blogs disponíveis na Web.

Os autores Rodrigues e Moreira (2012) fazem uma analogia dos métodos tradicionais de classificação com a Folksonomia afirmando que nos processos de indexação convencional, o usuário não é considerado como um participante ativo. Entretanto, a garantia do usuário é baseada no pressuposto de que ele é protagonista que subsidia ações para aperfeiçoar o processo de análise de assunto fazendo com que ele não seja esquecido nesse processo. De outro modo disposto, no âmbito dos sistemas que adotam a Folksonomia, o usuário é quem assegura essa garantia, além de ter a tarefa de representar os recursos.

Sendo assim, por meio da indexação colaborativa é possível integrar uma rede de associações baseadas nos descritores eleitos, individualmente ou por senso comum, independentemente do seu contexto de criação. Nessa perspectiva, a utilização das etiquetas varia entre o grupo de pessoas que as utilizam e os sistemas em que elas estão inseridas, fazendo com que sejam responsáveis pela representação dos objetos informacionais facilitando ou não a sua posterior recuperação por 
outro usuário. Isso se deve ao fato de que nem sempre as tags são atribuídas com o intuito de serem empregadas por outras pessoas em um determinado sistema, pois na maioria das vezes o usuário pretende apenas organizar os seus próprios dados (SANTOS, 2013).

González (2006) apresenta em sua pesquisa as características básicas inerentes à Folksonomia, dentre elas:

a) simplicidade no processo de representação dos recursos;

b) os termos elencados pelos usuários não são estruturados e disponíveis em uma hierarquia de conceitos (como os instrumentos de controle terminológico), o que os tornam mais flexíveis e possibilita a navegação por etiquetas relacionadas;

c) as tags refletem a dinamicidade da língua e permitem que haja coexistência de diversos pontos de vista dos usuários a respeito de um determinado recurso indexado.

Golder e Huberman (2006) realizaram uma pesquisa que objetivou analisar a dinâmica do uso das tags no site Delicious ${ }^{3}$. Como resultado de seus estudos, esses autores propõem uma classificação de etiquetas que se caracterizam como Folksonomia, baseando-se na função que cada tag cumpre em relação ao recurso indexado pelos usuários. Assim, para esses autores, a presença de um tipo ou outro de etiqueta define o tipo de Folksonomia existente em um determinado ambiente colaborativo. A classificação proposta pelos autores e que designa as funções das etiquetas analisadas é a seguinte:

a) etiquetas que identificam o assunto ou conteúdo do item categorizado;

b) tags que identificam qual o tipo de recurso (por exemplo, livro, blog, artigo, etc.);

c) etiquetas que identificam quem é o autor do recurso;

d) descritores que funcionam como categorias específicas, ou seja, que não podem ser empregados isoladamente, pois enriquecem outras tags para refinar ou especificar a descrição do recurso (por exemplo: banco - instituição financeira);

e) etiquetas que identificam propriedades ou características dos recursos mediante adjetivos como: "engraçado", "inspirador", "interessante" ou qualquer outro adjetivo que reflita a opinião do usuário no que concerne ao conteúdo indexado.

f) tags que demarcam a relação do recurso indexado com os usuários e funcionam como auto referência para os mesmos. Geralmente essas etiquetas iniciam com os

\footnotetext{
${ }^{3}$ Disponível em: <https://delicious.com/>. Acesso em: 12 maio 2018.
} 


\section{pronomes possessivos "meu", "minha" (por exemplo: "meus comentários", "meu conteúdo").}

Em consonância de evidências, Wal (2005) afirma que os usuários atribuem etiquetas que usam e acreditam que entendem objetivando 0 uso ou reuso das mesmas para a representação e recuperação da informação. Outrossim, no que concerne às características das tags na descrição de conteúdos Catarino e Baptista (2009) destacam que "as etiquetas são grafadas indistintamente em diferentes alfabetos e idiomas, na sua forma simples ou composta, singular ou plural, além de divergentes grafias".

No intuito de dar segmento a essas considerações, a seguir são apresentados os procedimentos metodológicos empreendidos na realização desta pesquisa.

\section{Procedimentos metodológicos}

Quanto aos objetivos a serem alcançados neste estudo, realizou-se uma pesquisa exploratória, uma vez que este trabalho buscou levantar como a Folksonomia vem sendo conceituada pelos pesquisadores da área de Ciência da Informação, no intuito de criar uma síntese sobre as definições do termo Folksonomia na literatura da área, bem como evidenciar os conceitos a ele relacionados.

Ademais, no que diz respeito à abordagem do problema, foi feita uma pesquisa bibliográfica, com foco na análise da literatura científica nacional e internacional sobre Folksonomia.

Mais especificamente, foram analisadas as definições extraídas dos trabalhos avaliados nesta pesquisa sobre o conceito de Folksonomia. Utilizou-se o método da análise de conteúdo fundamentado em Bardin (2009) como procedimento para analisar o conteúdo integral das produções científicas - possibilitando identificar, por meio da análise da literatura recuperada sobre a temática, as definições de Folksonomia elencadas nas pesquisas analisadas.

Para a realização a análise supracitada foi delimitado o domínio da Ciência da Informação, bem como foram selecionados artigos de periódicos, trabalhos publicados em anais de eventos técnico-científicos, Teses e Dissertações sobre a temática, refletindo a literatura sobre o tema em questão nessa área do conhecimento.

A coleta de tais produções científicas foi realizada em outubro de 2015 no Portal de Periódicos CAPES e nas seguintes bases de dados: Base de Dados Referencial de Artigos de Periódicos em Ciência da Informação (BRAPCI) e PERI (as quais indexam artigos de periódicos e trabalhos publicados em anais de eventos técnico-científicos na área de Ciência da Informação). Outrossim, esse processo de busca também foi realizado na Biblioteca Digital de Teses e Dissertações (BDTD) no intuito de também recuperar Teses e Dissertações sobre Folksonomia defendidas nos Programas de Pós-Graduação em Ciência da Informação do Brasil. 
Como estratégias de busca para a recuperação da produção científica nas bases de dados citadas anteriormente, foi delimitado o idioma inglês e português no intuito de recuperar os trabalhos publicados em âmbito nacional e internacional. Sendo assim, foi empregado na busca os termos "Folksonomia" e "Folksonomy", bem como as suas sucessivas variações terminológicas/conceituais como "classificação social", "social rank", "classificação popular", "popular rank", "etiquetagem colaborativa", "social tagging", "representação colaborativa da informação", "collaborative representation of information", "indexação social", "social indexing" nos campos assunto, título, resumo e palavras-chave dessas fontes de informação.

É importante ressaltar que alguns trabalhos recuperados (mediante as estratégias elencadas anteriormente) foram excluídos da análise, pois abordavam a Folksonomia na perspectiva da Ciência da Computação por pesquisadores dessa área do conhecimento - não fazendo parte da literatura da Ciência da Informação.

Nesse entendimento, o corpus final para a realização da análise da produção científica sobre Folksonomia foi constituído de sessenta e seis produções científicas, sendo elas: sete dissertações, duas teses, quarenta e nove artigos de periódicos e oito trabalhos publicados em anais de eventos técnico-científicos da área de Ciência da Informação. Sendo assim, no momento das buscas nas bases de dados supracitadas não foi delimitado o período de publicação dos trabalhos recuperados, possibilitando que as produções científicas pudessem ser identificadas e recuperadas em sua totalidade. Nesse sentido, verificou-se que os trabalhos recuperados estão distribuídos num intervalo de tempo de 2004 a 2014.

Após a listagem dos trabalhos recuperados, foi realizada uma leitura integral do conteúdo dessas produções científicas no intuito de identificar quais deles apresentavam os conceitos de Folksonomia. Em seguida, tais definições foram identificadas e extraídas no intuito de analisar quais os principais aspectos apresentados em cada uma delas, bem como identificar em que perspectiva cada trabalho analisado aborda o conceito de Folksonomia, baseando-se na técnica de análise de conteúdo de Bardin (2009).

Após a realização desses procedimentos, verificou-se que quinze produções científicas apresentaram o conceito de Folksonomia em seu conteúdo. Dessa forma, foi elaborado um quadro com as definições desse termo citando diretamente os seus respectivos autores.

A próxima seção contempla os resultados do estudo mediante análise e apresentação dos diversos usos do termo Folksonomia na literatura da Ciência da Informação através dos conceitos enunciados.

\section{Análise e síntese das definições do termo "Folksonomia"}

No contexto da Web social, a Folksonomia se apresenta como nova interface entre usuários e informação em rede. De acordo com Amstel 
(2007) ela faz parte de uma terminologia cunhada por Thomas Vander Wal numa lista de discussão sobre arquitetura da informação em 2004. Na lista de discussão, Eric Sheid propôs o termo "folkclassification" e Thomas Wander Wal complementou com "folksonomy", formado do termo "folk" do germânico "povo" e "taxonomy", do grego "ciência ou técnica de classificação".

Wal (2005), criador dessa terminologia, compreende a Folksonomia como o resultado da etiquetagem livre (descrição, representação) de objetos digitais no âmbito da Web pelos usuários. Para esse autor, por meio dessa indexação colaborativa o usuário realiza a representação da informação proporcionando um meio para conectar itens informacionais em meio digital. Assim, a Folksonomia é uma antítese ao termo taxonomia ${ }^{4}$ tendo como principal característica a criação de tags com função de descritor a partir da linguagem dos usuários. Ao invés de utilizar uma forma hierárquica e centralizada de categorização de alguma coisa, o usuário escolhe palavras-chave (conhecidas como "tags" ${ }^{5}$ ) para classificar a informação.

Levando em consideração que os estudos sobre Folksonomia são considerados recentes no âmbito da produção científica nacional e internacional, Catarino e Baptista (2007) mencionam em sua pesquisa que ainda não há uma conceituação única e padronizada do seu real significado. Assim, essas autoras apresentam algumas definições encontradas nas produções intelectuais de autores como Wal (2005), Mathes (2004), Sturtz (2004), Trant (2006) e Lund et al. (2005), afirmando em seus estudos que tais autores designavam a Folksonomia como um sistema de classificação, uma metodologia, abordagem ou processo de etiquetagem.

Haja vista que ainda não há uma conceituação única do real significado da Folksonomia na produção científica sobre essa temática, verificou-se a importância de criar uma síntese sobre os diversos usos desse termo, bem como os conceitos relacionados. Assim, elaborou-se um quadro cujas definições de Folksonomia foram extraídas diretamente dos trabalhos nacionais e internacionais da área de Ciência da Informação que a conceituavam. Na oportunidade, são apresentadas as principais acepções evidenciadas em cada conceito através dos elementos/termos presentes nas definições. É importante ressaltar que os conceitos estão organizados de maneira cronológica e as definições dos trabalhos da literatura internacional foram traduzidas no Quadro 1.

Quadro 1 - Definições de Folksonomia na literatura científica

\begin{tabular}{c|c}
\hline DEFINIÇÃO & ACEPÇÕES EVIDENCIADAS NO CONCEITO \\
\hline \hline $\begin{array}{c}\text { A Folksonomia é um conjunto de termos que um grupo de usuários } \\
\text { utilizou para etiquetar os conteúdos de recursos digitais da Web } \\
\text { (MATHES, 2004, online, tradução nossa). }\end{array}$ & *Contexto (web); \\
& *Ação (etiquetagem); \\
*Produto (conjunto de termos)
\end{tabular}

\footnotetext{
4 Estudo da classificação das coisas. É o ato de dar nomes, classificar, identificar.

${ }^{5}$ Em português significa "etiqueta", "identificação". "Taggear" é identificar, etiquetar alguma coisa.
} 
A Folksonomia é o conjunto de tags com uma ou mais palavras-chave em que os usuários, em um sistema compartilhado de gerenciamento de conteúdo, aplicam a conteúdos específicos (STURTZ, 2004, online, tradução nossa).
*Contexto (sistema compartilhado de gerenciamento de

conteúdo);

*Agente (usuários);

*Produto (conjunto de tags).
A Folksonomia é o resultado da etiquetagem livre de objetos digitais
no âmbito da Web pelos usuários (WAL, 2005, online, tradução nossa)

A Folksonomia é um vocabulário, ou lista de termos, que surge da sobreposição de etiquetas definidas por vários usuários ao marcar seus links favoritos ou marcadores para posterior recuperação (LUND et al., 2005, online, tradução nossa). Trata-se de um sistema que administra etiquetas atribuídas pelos
usuários aos recursos informacionais por eles indexados (OHMUKAI;
HAMASAKI; TAKEDA, 2005, online, tradução nossa).

*

\section{Uma nova abordagem emergente para a categorização distribuída de
recursos digitais (QUINTARELLI, 2005, online, tradução nossa). \\ As Folksonomias são sistemas de classificação distribuídos, criados por usuários individuais (GUY; TONKIN, 2006, online, tradução nossa).}
A Folksonomia consiste em uma classificação popular que é proveniente das ações de representação da informação as quais são realizadas por usuários de diversos serviços disponíveis na Web atual (SMITH, 2006, online, tradução nossa).
A Folksonomia, ao contrário da taxonomia, que se baseia da organização das informações por especialistas através de um vocabulário controlado, seria uma classificação social de "baixo para cima" (AQUINO, 2007, p. 11).

*Contexto (Web);

*Agente (usuários);

*Ação (etiquetagem); *Objeto (objetos digitais);

*Agente (usuários);

*Ação (marcação);

*Objeto (links favoritos ou marcadores)

*Produto (vocabulário ou lista de termos);

*Finalidade (posterior recuperação)

*Agente (usuários);

*Ação (indexação);

*Objeto (recursos informacionais);

*Produto (etiquetas)

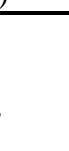

$\begin{aligned} & \text { *Ação (categorização) } \\ & \text { *Objeto (recursos digitais) }\end{aligned}$

*Objeto (recursos digitais)

*Agente (usuários individuais);

*Produto (sistema de classificação)

*Contexto (web)

*Método (classificação popular);

*Agente (usuários de diversos serviços);

*Ação (representação da informação).

*Método (classificação social); 
A partir de tais conceituações depreende-se que não há um consenso conceitual e terminológico acerca da Folksonomia haja vista que em alguns momentos ela é considerada pelos autores como um fenômeno; uma inovação; um sistema; uma classificação; um vocabulário; um método ou até mesmo um resultado de um processo conforme pode ser visualizado no Quadro 1. Além disso, é notável o emprego do termo tanto no singular ("Folksonomia") como no plural ("Folksonomias"), bem como a utilização dos sinônimos "classificação social", "classificação popular", "etiquetagem colaborativa", "indexação social", "representação colaborativa da informação" para fazer referência a essa palavra.

Em nítido contraste, Merholz (2004) não considera adequado o uso do termo Folksonomia, uma vez que considera que ele está erroneamente associado à taxonomia, afirmando que o termo apropriado seria "ethnoclassification".

Mediante os conceitos apresentados anteriormente no Quadro 1, através da realização da análise de conteúdo foi possível identificar sete aspectos que designam os principais elementos presentes nas definições de Folksonomia, são eles: "contexto" (ambiência em que a Foksonomia pode ser aplicada), "método" (técnica empregada para a criação da Folksonomia), "agente" (indivíduos que podem participar do processo de etiquetagem) , "ação" (atividade realizada para fins de criação da Folksonomia), "objeto" (recurso informacional que pode ser representado), "produto" (resultado gerado através do emprego da Folksonomia) e "finalidade" (objetivos da Folksonomia). Esses aspectos são apresentados no Quadro 2. 
Quadro 2 - Aspectos relacionados às definições de Folksonomia

\begin{tabular}{|c|c|c|c|c|c|c|}
\hline Contexto & Método & Agente & Ação & Objeto & Produto & Finalidade \\
\hline $\begin{array}{c}\text { Web } \\
\text { Sistema } \\
\text { compartilhado } \\
\text { Sistema } \\
\text { colaborativo } \\
\text { Redes sociais }\end{array}$ & $\begin{array}{c}\text { Etiquetagem } \\
\text { colaborativa } \\
\text { Classificação } \\
\text { popular } \\
\text { Classificação } \\
\text { social }\end{array}$ & $\begin{array}{c}\text { Povo } \\
\text { Usuários } \\
\text { Indivíduos } \\
\text { Comunidade }\end{array}$ & $\begin{array}{c}\text { Registro de } \\
\text { opiniões } \\
\text { Ações de } \\
\text { representação } \\
\text { Etiquetagem } \\
\text { Indexação } \\
\text { Categorização } \\
\text { Classificação } \\
\text { Organização } \\
\text { Marcação de links } \\
\text { favoritos } \\
\text { Compartilhamento } \\
\text { de recursos } \\
\text { informacionais }\end{array}$ & $\begin{array}{c}\text { Recursos ou } \\
\text { objetos } \\
\text { digitais na } \\
\text { Web } \\
\text { Links } \\
\text { favoritos } \\
\text { Textos } \\
\text { Imagens } \\
\text { Áudios } \\
\text { Vídeos } \\
\text { Produtos }\end{array}$ & $\begin{array}{c}\text { Conjunto de } \\
\text { termos } \\
\text { Conjunto de tags } \\
\text { Conjunto de } \\
\text { categorias } \\
\text { Coleção de tags } \\
\text { Vocabulário } \\
\text { Etiquetas } \\
\text { Termos } \\
\text { Conjunto de } \\
\text { metadados } \\
\text { Sistema de } \\
\text { classificação }\end{array}$ & $\begin{array}{l}\text { Representação da } \\
\text { Informação } \\
\text { Organização da } \\
\text { Informação } \\
\text { Posterior } \\
\text { recuperação }\end{array}$ \\
\hline
\end{tabular}

Fonte: Elaborado pelos autores. 
Em síntese, verifica-se que a maneira como o conceito de Folksonomia é interpretado nas produções científicas sobre esta temática no âmbito da Ciência da Informação está relacionada a um plano ou uma abordagem ampla, haja vista que são apresentadas várias discussões para defini-la levando em consideração as suas principais características. Portanto, infere-se que não há ainda uma definição única, clara, bem enunciada e rigorosa, cabendo outros estudos que visem aprofundar e ampliar discussões conceituais acerca dessa definição.

Objetivando contribuir com um passo na direção à consolidação do conceito de Folksonomia, levando-se em conta os conceitos elencados anteriormente e ratificando as ideias de Thomas Vander Wal (por ser o criador dessa terminologia), a Folksonomia pode ser conceituada nesta pesquisa como: o resultado do processo de etiquetagem livre (atribuição de etiquetas, palavras-chave) realizada pelos usuários mediante 0 emprego de termos provenientes de linguagem natural - dispensando o uso de vocabulários controlados- em ambientes digitais colaborativos visando indexar recursos informacionais compartilhados de qualquer formato (textos, imagens, áudio, vídeo etc.) para fins de sua representação e recuperação.

Nesse entendimento, observa-se que a diferença entre etiquetagem e Folksonomia consiste no fato de que a primeira se constitui no processo de atribuir etiquetas (descritores, palavras-chave) e a segunda é equivalente ao resultado desta atribuição.

\section{Considerações finais}

Através da análise realizada, observa-se que a Folksonomia está intimamente ligada à integração do usuário na representação da informação de uso pessoal e/ou coletivo em ambientes digitais, visando o compartilhamento e a recuperação de recursos.

Diante disso, no âmbito dos sistemas colaborativos que utilizam a indexação colaborativa, o usuário escolhe descritores livres ou palavraschave para etiquetar um recurso informacional. Além disso, observa-se que a diferença entre a etiquetagem e a Folksonomia consiste no fato de que a primeira se constitui no processo de atribuir etiquetas (descritores, palavras-chave) e a segunda é equivalente ao resultado desta atribuição.

Portanto, pode-se afirmar que os objetivos propostos neste estudo foram alcançados uma vez que foi criada e apresentada uma síntese das definições do termo Folksonomia na literatura da Ciência da Informação, bem como os conceitos relacionados. Ademais, foram identificados sete aspectos que designam os principais elementos presentes nas definições de Folksonomia, os quais são: "contexto", "método", "agente", "ação", "objeto", "produto" e "finalidade".

Outrossim, objetivando contribuir com um passo na direção à consolidação do conceito do termo Folksonomia levando em conta as definições extraídas dos trabalhos analisados na pesquisa, foi apresentada uma definição para o termo em questão. Sendo assim, espera-se que 
esta pesquisa contribua para o entendimento do que é a Folksonomia e os demais termos relacionados.

Como pesquisa futura para ampliar os aspectos apresentados neste trabalho será realizada uma nova busca e análise das definições com vistas a criar um mapa conceitual evidenciando os conceitos de Folksonomia apresentados nos trabalhos e as interligações das definições de modo esquematizado. Adicionalmente, os procedimentos metodológicos aqui adotados serão expandidos visando a ampliação do escopo de análise dos conceitos de Folksonomia apresentados nas produções científicas oriundas de qualquer área do conhecimento, sem que seja delimitado o campo da Ciência da Informação.

\section{Referências}

AMSTEL, V. F. Folksonomia: vocabulário descontrolado na arquitetura da informação ou samba do criolo doido. 2007. Disponível em: <http://www.guilhermo.com/ai_biblioteca/referencia.asp?referencia $=317$ $>$. Acesso em: 20 maio 2018.

AQUINO, M. C. A potencialização da memória coletiva através do hipertexto na Web 2.0. In: CONGRESSO BRASILEIRO DE CIÊNCIAS DA COMUNICAÇÃO, 30., 2007, Santos. Anais... Santos: Sociedade Brasileira de Estudos interdisciplinares da Comunicação, 2007. p. 1-16.

ASSIS, J. de; MOURA, M. A. Folksonomia: a linguagem das tags. Encontros bibli: revista eletrônica de Biblioteconomia e Ciência da Informação, v. 18, n. 36, p. 85-106, jan./abr. 2013.

BARDIN, L. Análise de conteúdo. Lisboa; Portugal: Edições 70; LDA, 2009.

BLATTMANN, U.; SILVA, F. C. C. da. Colaboração e interação na Web 2.0 e biblioteca 2.0. Revista ACB: biblioteconomia em Santa Catarina, Florianópolis, v. 12, n. 2, p. 191-215, jul./dez. 2007. Disponível em: <http://revista.acbsc.org.br/racb/article/view/530/664>. Acesso em: 10 ago. 2017.

BRANDT, M.; MEDEIROS, M. B. B. Folksonomia: esquema de representação do conhecimento? Transinformação, Campinas, v. 22, n. 2, p. 111-121, maio/ago. 2010.

CALDAS, W. F.; MOREIRA, M. P. Folksonomia e classificação de etiquetas: estudo de caso Flickr. 2009. Disponível em: <http://repositorios.questoesemrede.uff.br/repositorios/bitstream/handle/ 123456789/62/GT\%202\%20Txt\%2010-

$\% 20$ CALDAS $\% 2 c \% 20$ Wagner\%20F..pdf?sequence=1>. Acesso em: 15 ago. 2017.

CATARINO, M.; BAPTISTA, A. Folksonomia: um novo conceito para a organização dos recursos digitais na web. DataGramaZero, Rio de Janeiro, v. 8, n. 3, 2007. 
CATARINO, M.; BAPTISTA, A. Folksonomias: características das etiquetas na descrição de recursos da web. Inf. Inf., Londrina, v. 14, n. esp., p. 46$67,2009$.

GOLDER, S.A.; HUBERMAN, B.A. The structure of collaborative tagging systems. $2006 . \quad$ Disponível em: <http://www.hpl.hp.com/research/idl/papers/tags/tags.pdf>. Acesso em: 25 maio 2018.

GONZÁLEZ J. A. M. La representación y recuperación de los contenidos digitales: de los tesauros conceptuales a las folksonomías. In: TRAMULLAS, J. (Coord.). Tendencias en documentación digital. Gijón: Trea, 2006. p. 81-108.

GUY, M.; TONKIN, E. Folksonomies: tidying up tags? D-Lib Magazine, v. 12, $\mathrm{n}$. 1 2006. Disponível em: <http://www.dlib.org/dlib/january06/guy/01guy.html>. Acesso em: 16 jun. 2018.

LUND, B. et al. Social Bookmarking Tools (II): a case study: Connotea. DLib Magazine, v. 11, n. 4, 2005. Disponível em: <http://www.dlib.org/dlib/april05/lund/04lund.html>. Acesso em: 24 jul. 2017.

MATHES, A. Folksonomies: cooperative classification and communication through shared metadata. 2004. Disponível em: $<$ http://www.adammathes.com/academic/computermediatedcommunication/folksonomies.pdf>. Acesso em: 5 jul. 2017.

MERHOLZ, P. Metadata for the masses. 2004. Disponível em: <http://www.adaptivepath.com/ideas/e000361/>. Acesso em: 23 set. 2017.

OHMUKAI, I.; HAMASAKI, M.; TAKEDA, H. A proposal of community-based folksonomy with rdf metadata. In: INTERNATIONAL SEMANTIC WEB CONFERENCE, 5., Galeway, Ireland. Anais... Gaway; Ireland: ISWC, 2005. p. 1-8. Disponível em: <http://wwwkasm.nii.ac.jp/papers/takeda/05/ohmukai05iswc.pdf>. Acesso em: 26 out. 2017.

QUINTARELLI, E. Folksonomies: power to the people. In: INCONTRO ISKO ITALIA - UNIMIB, Milan, 2005. Anais... Milan: ISKO ITALIA, 2005. Disponível em: <http://www.iskoi.org/doc/folksonomies.htm>. Acesso em: 15 jul. 2017.

ROCHA, L. Folksonomia: a classificação de conteúdo na web em nossas mãos. Revista WebDesign, v. 31, p. 22-35, jul. 2007. Disponível em: <http://www.arteccom.com.br/webdesign/downloads/31/1.pdf>. Acesso em: 10 out. 2014.

RODRIGUES, A. A. de A.; MOREIRA, M. P. Folksonomia: análise de etiquetagem de imagens no Flickr. Informe: Estudos em Biblioteconomia e Gestão da Informação, Recife, v. 1, n. 1, p. 87-101, 2012. 
SANTINI, R. M.; SOUZA, R. F. Classificação colaborativa de conteúdos não-textuais na internet: as novas formas de mediação e organização da informação da música através da Folksonomia. In: ENCONTRO NACIONAL DE PESQUISA EM CIÊNCA DA INFORMAÇÃO, 11., 2010. Rio de Janeiro. Anais... Rio de Janeiro: Associação Nacional de Pesquisa e Pós-Graduação em Ciência da Informação, 2010. p. 1-24.

SANTOS, H. P. Etiquetagem e Folksonomia: o usuário e sua motivação para organizar e compartilhar informação na web 2.0. Perspectivas em Ciência da Informação, v. 1, n. 2, p. 91-104, abr./jun. 2013.

SMITH, M. K. Viewer tagging in art museums: comparisons to concepts and vocabularies of art museum visitors. In: SIG/CR CLASSIFICATION RESEARCH WORKSHOP, 17., Austin, USA, 2006. Papers... Austin: American Society for Informationm Science and Technology, 2006. p. 119. Disponível em: <http://www.slais.ubc.ca/users/sigcr/ sigcr06smith.pdf>. Acesso em: 6 set. 2017.

STREHL, L. As Folksonomias entre os conceitos e os pontos de acesso: as funções de descritores, citações e marcadores nos sistemas de recuperação da informação. Perspectivas em Ciência da Informação, v. 16, n. 2, p. 101-114, jun./ago. 2011.

STURTZ, D. N. Communal categorization: the folksonomy. 2004. Disponível em:<http://www.davidsturtz.com/drexel/622/communalcategorization-the-folksonomy.html>.Acesso em: 7 out. 2017.

TRANT, J. Exploring the potential for social tagging and folksonomy in art museums: proff of concept. New Review of Hypermedia and Multimedia, V. 12, n. 1, p. 63-81, 2006.

VIERA, A. F. G.; GARRIDO, I. dos S. Folksonomia como uma estratégia para recuperação colaborativa da informação. DataGramaZero - Revista de Ciência da Informação, v. 12, n. 2, abr. 2011.

WAL, T. V. Folksonomy definition and wikipedia. 2005. Disponível em: <http://www.vanderwal.net/random/entrysel.php?blog=1750>. Acesso em: 18 set. 2014 .

WAL, T. V. Online information folksonomy presentation posted. Personal Infoclud. $2006 . \quad$ Disponível em: <http://www.personalinfocloud.com/blog?category=Folksonomy $>$. Acesso em: 27 jul. 2017. 